\title{
Growth of Multinary Copper-Based Sulfide Shells on CuInSe Nanocrystals for Significant Improvement of Their Near-Infrared Emission
}

Jiajia Ning, ${ }^{\dagger}$ Yuan Xiong, ${ }^{\ddagger}$ Fei Huang,,$\$$ Zonghui Duan, ${ }^{\dagger}$ Stephen V. Kershaw ${ }^{\dagger}$ and Andrey L. Rogach ${ }^{\dagger}, *$

$\dagger$ Department of Materials Science, and Engineering and Centre of Functional Photonics, City University of Hong Kong, Hong Kong SAR, China

¥Department of Chemistry, City University of Hong Kong, Hong Kong SAR, China

\# Department of Physics, City University of Hong Kong, Hong Kong SAR, China

${ }^{\$}$ Institute for Advanced Materials and Technology, University of Science and Technology Beijing, Beijing 100083, China

Corresponding Author: andrey.rogach@,cityu.edu.hk

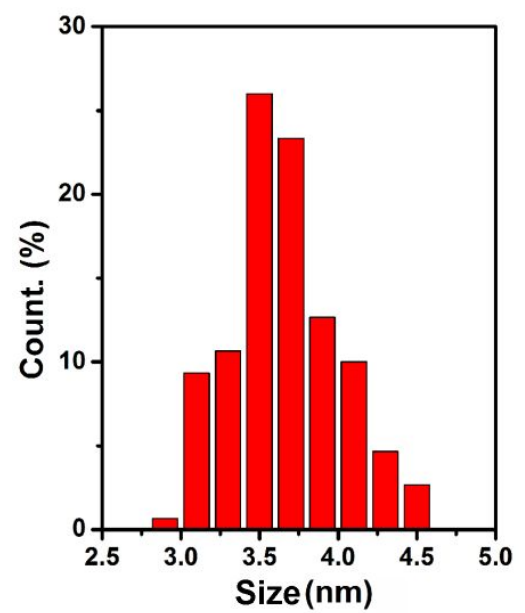

Figure S1. Size histogram of the CISe core NCs. 


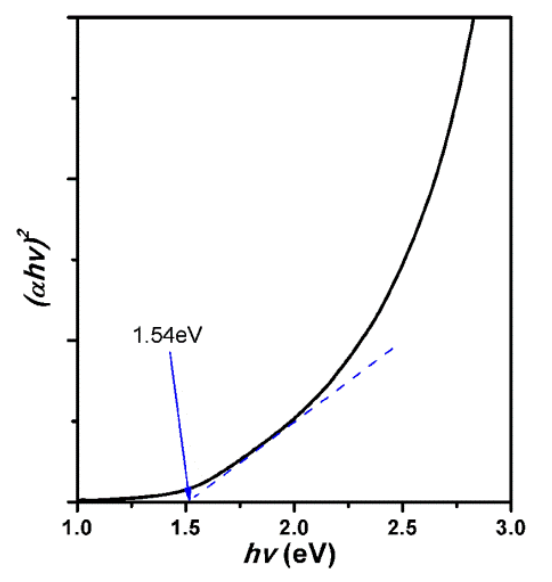

Figure S2. Estimation of the band gap of the CISe core NCs from the Tauc plot.

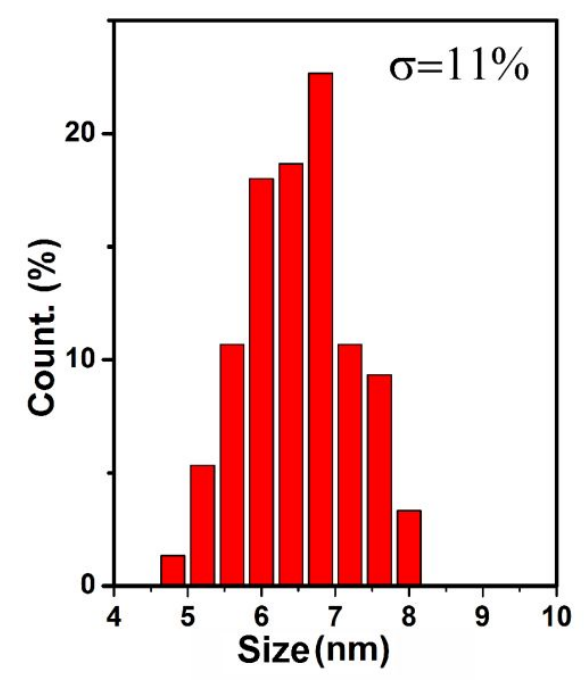

Figure S3. Size histogram of the CISe/CIS core/shell NCs.

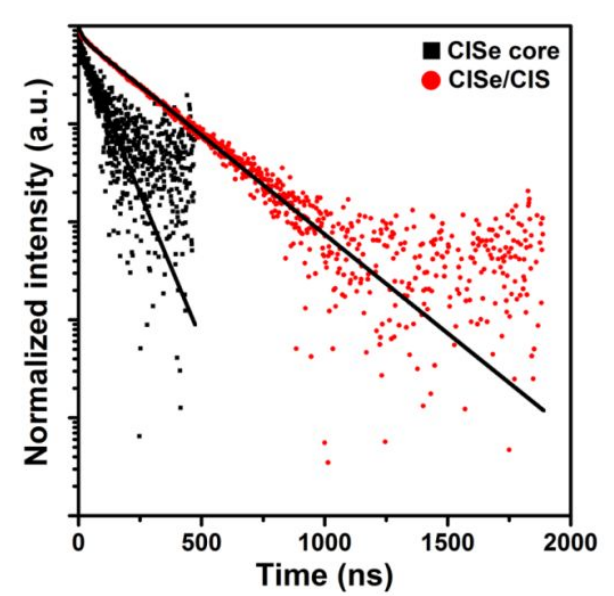

Figure S4. PL decays of the CISe core NCs (black squares) and CISe/CIS core/shell NCs (red spheres). Solid lines represent two-exponential fits. 


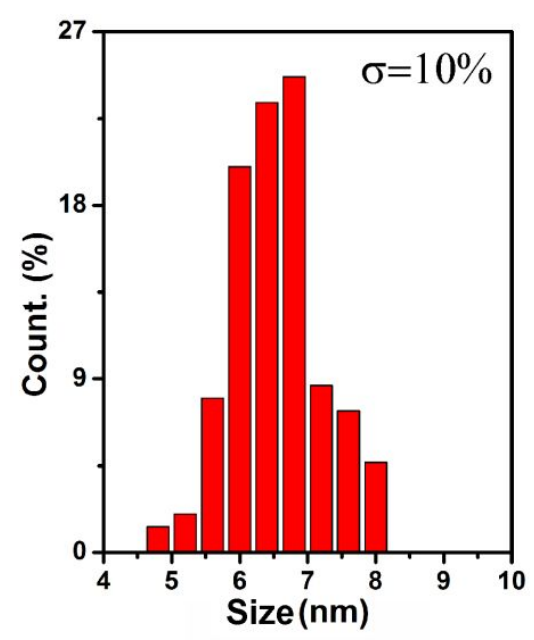

Figure S5. Size histogram of the CISe/CIZS core/shell NCs.

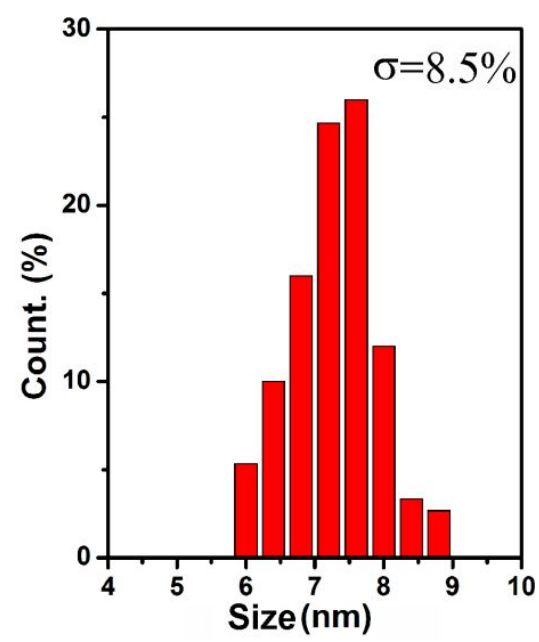

Figure S6. Size histogram of the CISe/CISSe core/shell NCs.

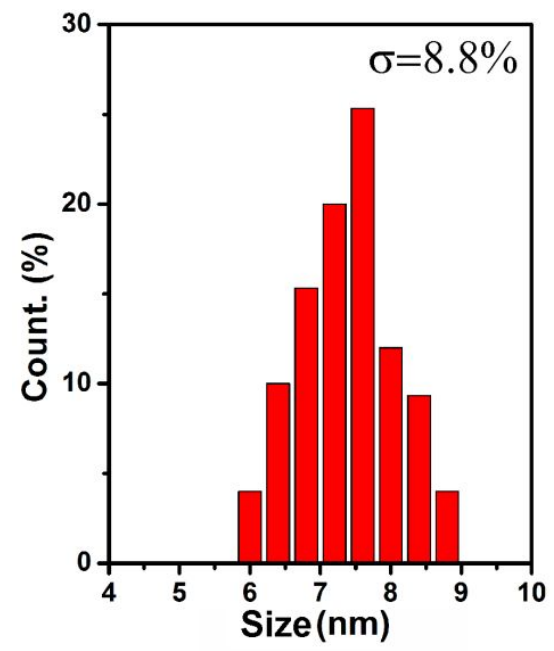

Figure S7. Size histogram of the CISe/CIZSSe core/shell NCs. 